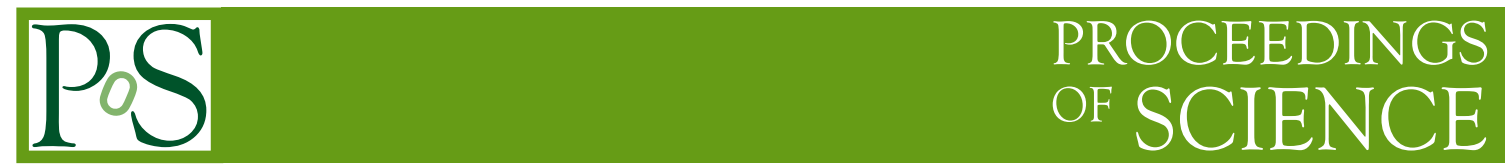

\title{
Will Planck Observe Gravity Waves?
}

\author{
Qaisar Shafi*i \\ Bartol Research Institute, Department of Physics and Astronomy, University of Delaware \\ 217 Sharp Lab Newark, DE 19716, USA \\ E-mail: shafiebartol.udel.edu
}

\begin{abstract}
Assume that inflation is driven by a gauge singlet scalar field with a Higgs potential. The tensor to scalar ratio $\mathrm{r}$, a canonical measure of gravity waves generated during inflation, turns out in this case to be $\gtrsim 0.02$, provided the scalar spectral index $n_{s} \geq 0.96$. Thus, Planck or some other comparable experiment should observe $r$ sooner or later.

In minimal supersymmetric hybrid inflation, on the other hand, $r \sim 10^{-10}$, and therefore lies well below the reach of Planck and any foreseeable experiment. Observable $r$ values, $\sim 0.02$ or so, are realized in non-minimal versions of supersymmetric hybrid inflation.
\end{abstract}

The European Physical Society Conference on High Energy Physics -EPS-HEP2013

18-24 July 2013

Stockholm, Sweden

\footnotetext{
${ }^{*}$ Speaker.

${ }^{\dagger}$ This work is supported in part by the DOE Grant No. DE-FG02-91ER40626.
} 


\section{Introduction}

The discovery of primordial gravity waves generated during inflation would have far reaching consequences both for particle physics and cosmology. A canonical measure for these gravity waves is provided by the tensor to scalar ratio $r$, which is related to the energy scale $V_{0}^{1 / 4}$ during inflation.

The Planck satellite experiment it appears can measure $r$ values as low as 0.03 , which corresponds to $V_{0}^{1 / 4} \sim 10^{16} \mathrm{GeV} \sim M_{\mathrm{GUT}}$, the scale of supersymmetric grand unification!

This explains why measuring $r$ is perhaps the greatest challenge for the Planck satellite and other experiments, since it would have dramatic implications both for cosmology and particle physics.

It is worth pointing out that with $r \leq 0.15$, according to Planck and WMAP 9 measurements, the vacuum energy scale during observable inflation is of order $M_{\mathrm{GUT}}$ or smaller, that is at least two orders of magnitude lower than the Planck scale of $2.4 \times 10^{18} \mathrm{GeV}$.

In the following discussion it is shown that if inflation is driven by a gauge singlet scalar field with a Higgs potential, there is a lower bound on the tensor to scalar ratio, namely $r_{\min } \sim 0.02$, provided the scalar spectral index $n_{s}$ is close to 0.96 . Thus, according to this (non-supersymmetric) inflationary model, Planck should observe gravity waves.

We then consider a model of supersymmetric hybrid inflation. The minimal version of this class of models predicts that $r \ll 1$, well below the reach of Planck or other foreseeable experiments. However, non-minimal versions of hybrid inflation predict $r$ values as large as 0.02 or so, and which are therefore testable by Planck.

The talk covers inflationary cosmology, tree level Higgs inflation and quantum smearing of tree level predictions, then supersymmetric Higgs (Hybrid) inflation. We conclude with a summary.

\section{Inflationary Cosmology}

A successful model of primordial inflation should explain the flatness and isotropy of the Universe, provide origin of $\delta T / T$, recover Hot Big Bang Cosmology, explain the baryon asymmetry, have a plausible cold dark matter (CDM) candidate and offer testable predictions for the spectral index $\left(n_{s}\right)$, the tensor to scalar ratio $(r)$ and the running of the spectral index $d n_{s} / d \ln k$.

\section{Slow-roll Inflation}

Inflation is driven by some potential $V(\phi)$, where $\phi$ denotes the inflaton field. The slow roll parameters $(\varepsilon, \eta)$, the spectral index $\left(n_{s}\right)$ and the tensor to scalar ratio $(r)$ are given by

$$
\varepsilon=\frac{m_{p}^{2}}{2}\left(\frac{V^{\prime}}{V}\right)^{2}, \quad \eta=m_{p}^{2}\left(\frac{V^{\prime \prime}}{V}\right), \quad n_{s}-1 \equiv \frac{d \ln \Delta_{\mathscr{R}}^{2}}{d \ln k}, \quad r \equiv \frac{\Delta_{h}^{2}}{\Delta_{\mathscr{R}}^{2}} .
$$

where $\Delta_{h}^{2}$ and $\Delta_{\mathscr{R}}^{2}$ are the spectra of primordial gravity waves and curvature perturbation respectively.

In the slow-roll approximation (i.e. $(\varepsilon,|\eta|) \ll 1), n_{s}$ and $r$ are given as 


$$
n_{s} \simeq 1-6 \varepsilon+2 \eta, \quad r \simeq 16 \varepsilon
$$

The tensor to scalar ratio $r$ is related to the energy scale of inflation via $V\left(\phi_{0}\right)^{1 / 4}=3.3 \times$ $10^{16} r^{1 / 4} \mathrm{GeV}$. The amplitude of the curvature perturbation and the spectrum of the tensor perturbation are given by

$$
\Delta_{\mathscr{R}}^{2}=\frac{1}{24 \pi^{2}}\left(\frac{V / m_{p}^{4}}{\varepsilon}\right)_{\phi=\phi_{0}}, \quad \Delta_{h}^{2}=\frac{2}{3 \pi^{2}}\left(\frac{V}{m_{P}^{4}}\right)_{\phi=\phi_{0}}
$$

The number of $e$-folds after the comoving scale $l_{0}=2 \pi / k_{0}$ has crossed the horizon is given by

$$
N_{0}=\frac{1}{m_{p}^{2}} \int_{\phi_{e}}^{\phi_{0}}\left(\frac{V}{V^{\prime}}\right) d \phi
$$

Inflation ends when $\max \left[\varepsilon\left(\phi_{e}\right),\left|\eta\left(\phi_{e}\right)\right|\right]=1$.

\section{Gauge Singlet Higgs Inflation}

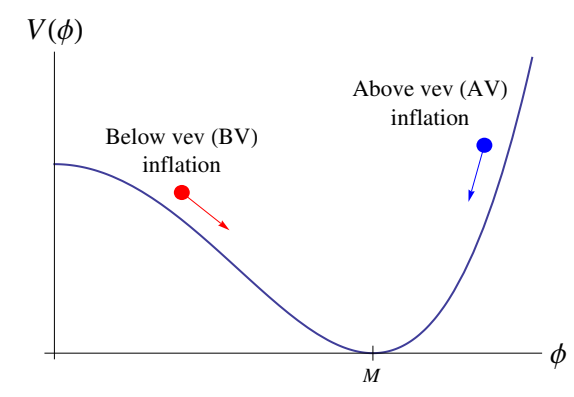

Figure 1: Higgs Potential

Consider the Higgs Potential:

$$
V(\phi)=V_{0}\left[1-\left(\frac{\phi}{M}\right)^{2}\right]^{2} \longleftarrow \text { (tree level) }
$$

where $\phi$ denotes a gauge singlet inflaton field.

The tree level results for the Higgs inflation model are presented in Fig.(2-a). Taking into quantum corrections, the potential becomes,

$$
V=\frac{1}{4} \lambda\left(\phi^{2}-\mathrm{v}^{2}\right)^{2}+\frac{C}{16 \pi^{2}} \phi^{4} \log \left[\frac{\phi}{\mathrm{v}}\right]+\text { const. }
$$

The corresponding results are shown in Fig.(2-b), including those for Coleman-Weinberg inflation [2]. 


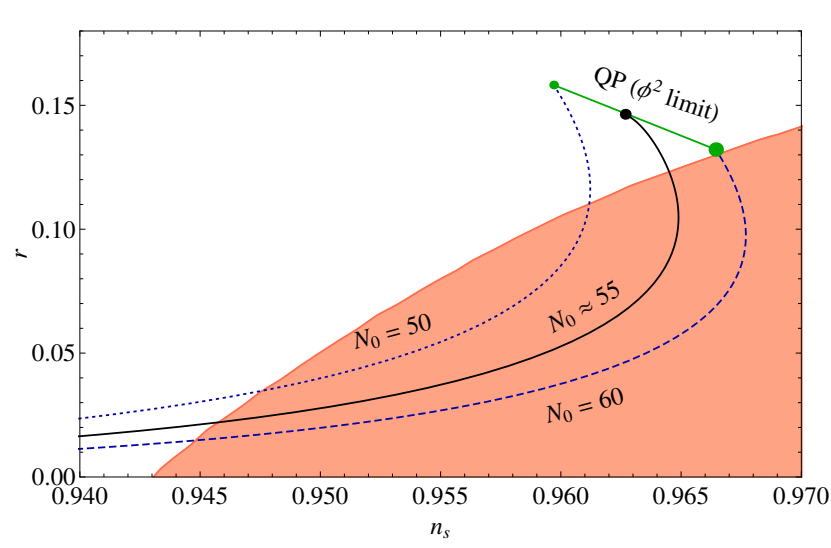

(a) Tree Level

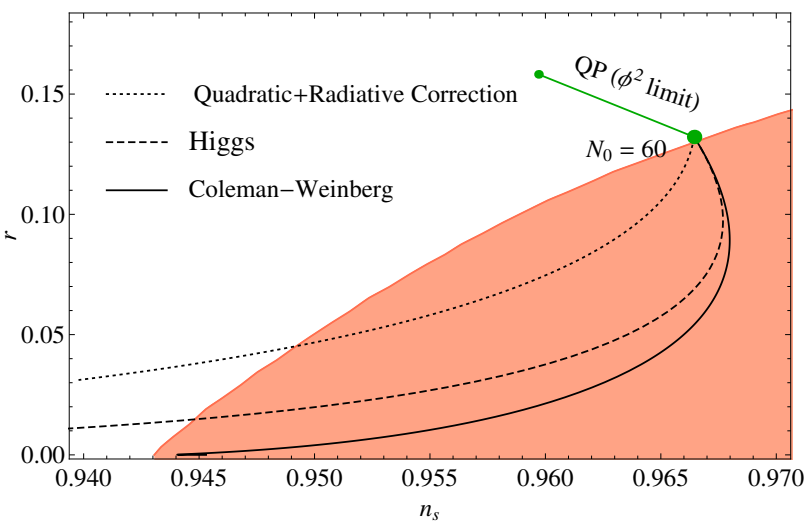

(b) Including Quantum Corrections

Figure 2: $n_{s}$ vs. $r$ for Higgs Inflation

\section{Motivation for SUSY}

- Resolution of the gauge hierarchy problem

- Predicts plethora of new particles which LHC should find

- Unification of the SM gauge couplings at $M_{\mathrm{GUT}} \sim 2 \times 10^{16} \mathrm{GeV}$.

- Cold Dark Matter candidate (Lightest Supersymmetric Particle)

- Radiative electroweak symmetry breaking

- String theory requires SUSY

\section{SUSY Hybrid Inflation}

- Attractive scenario in which inflation can be associated with suitable gauge symmetry breaking $G \longrightarrow H$

- Simplest inflation model is based on

$$
W=\kappa S\left(\Phi \bar{\Phi}-M^{2}\right)
$$

where $S$ is a gauge singlet superfield, $(\Phi, \bar{\Phi})$ belong to suitable representation of $G$.

- Need $\Phi, \bar{\Phi}$ pair in order to preserve SUSY while breaking $G \longrightarrow H$ at a scale $M \gg \mathrm{TeV}$.

- Imposing R-symmetry

$$
\Phi \bar{\Phi} \rightarrow \Phi \bar{\Phi}, S \rightarrow e^{i \alpha} S, W \rightarrow e^{i \alpha} W
$$

$W$ is a unique renormalizable superpotential. 


\section{7. $U(1)_{B-L}$ Hybrid Inflation}

At the renormalizable level the SM displays an "accidental" global $U(1)_{B-L}$ symmetry. If we "gauge" this symmetry so that $U(1)_{B-L}$ becomes a local symmetry, one may introduce $3 \mathrm{SM}$ singlet (right-handed) neutrinos in order to cancel the gauge anomalies. Cancelling anomalies with $3 \mathrm{SM}$ singlet neutrinos has several advantages:

- See-saw mechanism is automatic and neutrino oscillations can be understood.

- RH neutrinos acquire masses only after $U(1)_{B-L}$ is spontaneously broken. Neutrino oscillations require that $\mathrm{RH}$ neutrino masses are $\lesssim 10^{14} \mathrm{GeV}$.

- RH neutrinos can trigger leptogenesis after inflation, which subsequently gives rise to the observed baryon asymmetry;

- Last but not least, the presence of local $U(1)_{B-L}$ symmetry enables one to explain the origin of $Z_{2}$ 'matter' parity of MSSM. (It is contained in $U(1)_{B-L} \times U(1)_{Y}$, if $B-L$ is broken by a scalar VEV, with the scalar carrying two units of $B-L$ charge.)

The tree level potential is given by

$$
V_{F}=\kappa^{2}\left(M^{2}-\left|\Phi^{2}\right|\right)^{2}+2 \kappa^{2}|S|^{2}|\Phi|^{2}
$$

Including supergravity corrections and soft SUSY breaking terms, and employing the minimal Kähler potential,

$K=|S|^{2}+|\Phi|^{2}+|\bar{\Phi}|^{2}$,

the inflationary potential is given by [3]

$V \simeq \kappa^{2} M^{4}\left(1+\left(\frac{M}{m_{p}}\right)^{4} \frac{x^{4}}{2}+\frac{\kappa^{2} \cdot \mathscr{N}}{8 \pi^{2}} F(x)+a_{S}\left(\frac{m_{3 / 2} x}{\kappa M}\right)+\left(\frac{m_{3 / 2} x}{\kappa M}\right)^{2}\right)$,

with

$a_{s}=2|2-A| \cos [\arg S+\arg (2-A)], x=|S| / M$. $3 e)$.

The results for $n_{s}, r, \frac{d n_{s}}{d \ln k}$ and the supersymmetry breaking scale $M$ are summarized in Figs.(3a- 


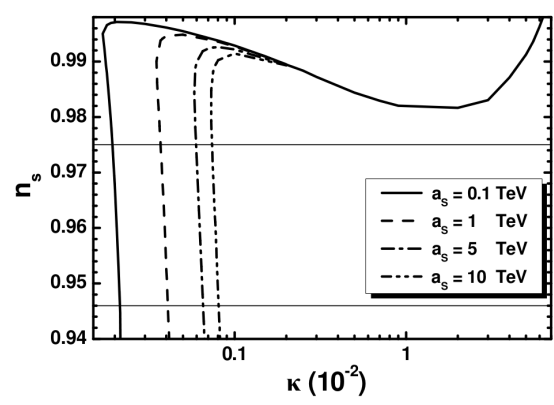

(a)

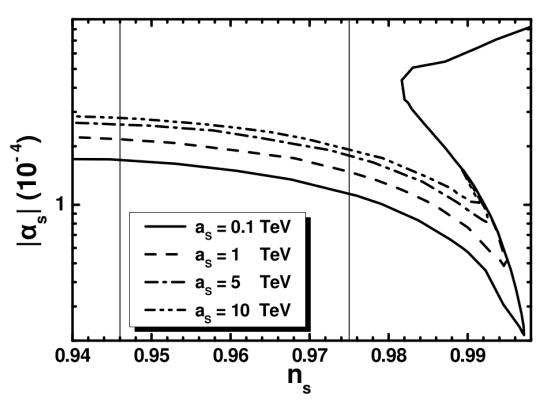

(c)

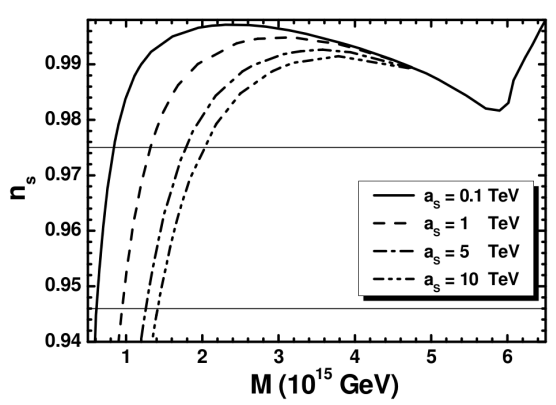

(b)

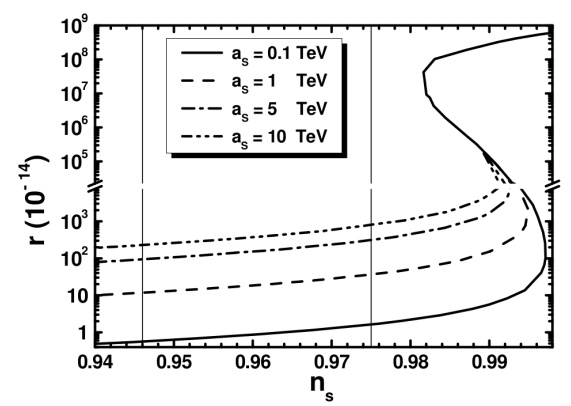

(d)

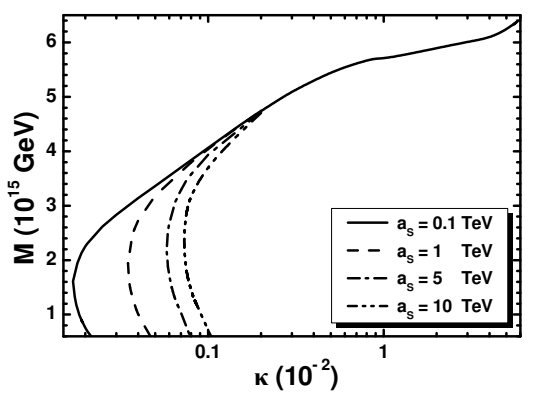

(e)

Figure 3: Results for $U(1)_{B-L}$ Hybrid Inflation

\section{Non-Minimal Hybrid Inflation}

With a non-minimal Kähler potential it is possible to realize $r$ values as large as 0.02 or so [4], which can be measured by Planck.

\section{Summary}

- The predictions of $r$ (primordial gravity waves) for various models of inflation are as follows:

- Gauge Singlet Higgs Inflation: 


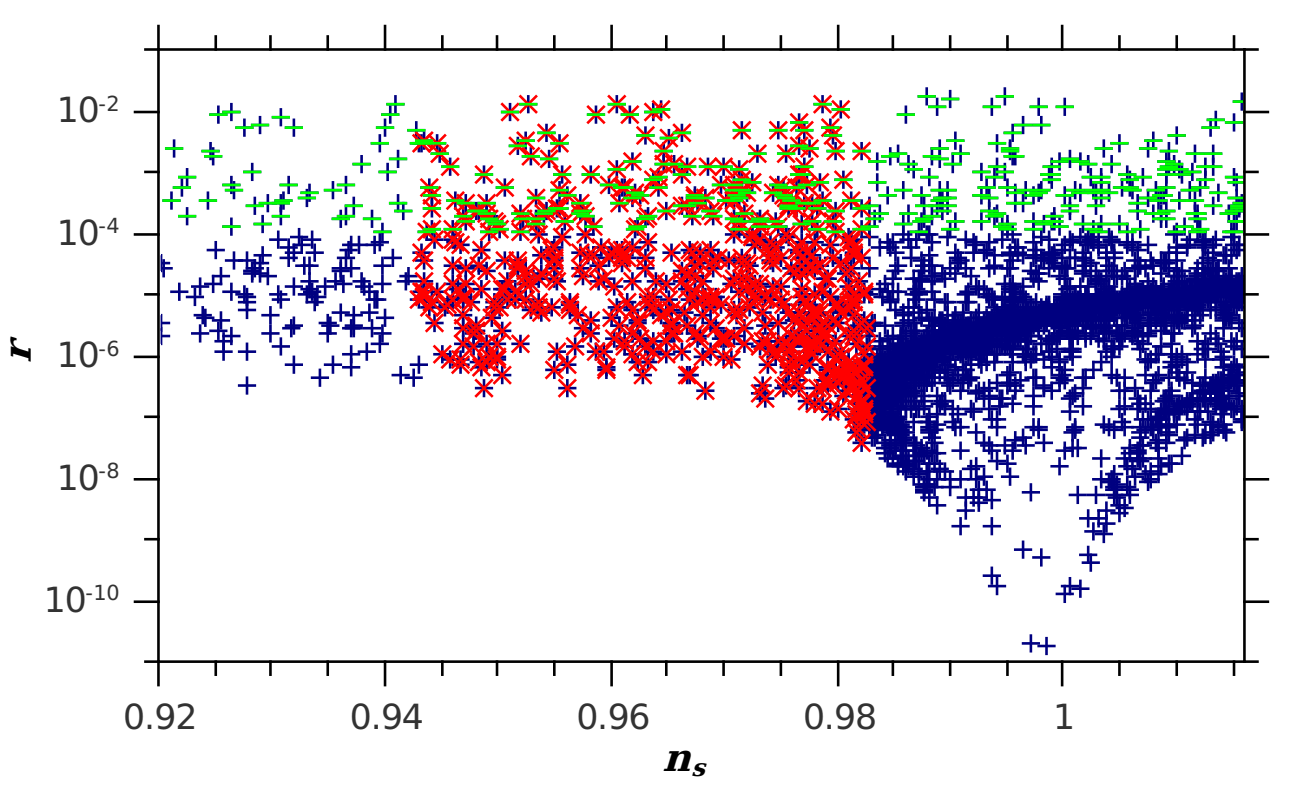

Figure 4: $r$ vs. $n_{s}$ (non-minimal SUSY hybrid inflation).

$$
r \gtrsim 0.02 \text { for } n_{s} \geq 0.96
$$

- SUSY Higgs (Hybrid) Inflation:

$$
r \lesssim 10^{-10} \text { (minimal), } \quad r \lesssim 0.02 \text { (non-minimal) }
$$

- Updated results for $r, \frac{d n_{s}}{d \ln k}, n_{s}$ are eagerly awaited.

\section{References}

[1] For Higgs Inflation: R. Kallosh and A. D. Linde, JCAP 0704, 017 (2007) [arXiv:0704.0647 [hep-th]]; M. U. Rehman, Q. Shafi and J. R. Wickman, Phys. Rev. D 78, 123516 (2008) [arXiv:0810.3625 [hep-ph]]; M. U. Rehman and Q. Shafi, Phys. Rev. D 81, 123525 (2010) [arXiv:1003.5915 [astro-ph.CO]].

[2] For Coleman-Weinberg Inflation: Q. Shafi and A. Vilenkin, Phys. Rev. Lett. 52, 691 (1984); Q. Shafi and V. N. Senoguz, Phys. Rev. D 73, 127301 (2006) [astro-ph/0603830];

M. U. Rehman, Q. Shafi and J. R. Wickman, Phys. Rev. D 79, 103503 (2009) [arXiv:0901.4345 [hep-ph]].

[3] For recent discussion of SUSY hybrid inflation: C. Pallis and Q. Shafi, Phys. Lett. B 725, 327 (2013) [arXiv:1304.5202 [hep-ph]]; R. Armillis and C. Pallis, Chapter in "Recent Advances in Cosmology", edited by A. Travena and B. Soren, Nova Science Publishers, Inc. [arXiv:1211.4011 [hep-ph]].

Also see: G. R. Dvali, Q. Shafi and R. K. Schaefer, Phys. Rev. Lett. 73, 1886 (1994) [hep-ph/9406319]; E. J. Copeland, A. R. Liddle, D. H. Lyth, E. D. Stewart and D. Wands, Phys. Rev. D 49, 6410 (1994) [astro-ph/9401011]; G. Lazarides, R. K. Schaefer and Q. Shafi, Phys. Rev. D 56, 1324 (1997) [hep-ph/9608256]; A. D. Linde and A. Riotto, Phys. Rev. D 56, 1841 (1997) [hep-ph/9703209]; V. N. Senoguz and Q. Shafi, Phys. Lett. B 596, 8 (2004) [hep-ph/0403294].

[4] M. Civiletti, M. U. Rehman, Q. Shafi and J. R. Wickman, Phys. Rev. D 84, 103505 (2011) [arXiv:1104.4143 [astro-ph.CO]]. 